\title{
Anpassung an Überschwemmungen in südostasiatischen Megastädten - Was kann der Privatsektor leisten?
}

\author{
Thomas Neise ${ }^{1}\left[\right.$ D $\cdot$ Roxana Leitold ${ }^{2}$ \\ Eingegangen: 10. Februar 2021 / Überarbeitet: 16. April 2021 / Angenommen: 19. April 2021 / Online publiziert: 4. Juni 2021 \\ (c) Der/die Autor(en) 2021
}

\section{Zusammenfassung}

Die fortschreitende Urbanisierung und erste Auswirkungen des Klimawandels verstärken das Überschwemmungsrisiko in küstennahen südostasiatischen Megastädten. Lokale Industriebetriebe sind insbesondere in ihrer Funktions- und Wettbewerbsfähigkeit betroffen. Gleichzeitig wird in den Debatten zu Klimawandelanpassung und Katastrophenrisikominderung die Beteiligung des Privatsektors an Flutschutzmaßnahmen diskutiert, da die lokalen Behörden in Südostasien das Flutrisiko häufig nicht eigenständig substanziell reduzieren können. Vor diesem Hintergrund wird untersucht, wie Industriebetriebe in Ho Chi Minh City (Vietnam) und Jakarta (Indonesien) auf Überschwemmungen reagieren, welche Anpassungsmaßnahmen sie durchführen und inwiefern ihr Engagement einen Beitrag zur kollektiven Anpassungen leistet. Die Analyse von 63 Tiefeninterviews mit Industriebetrieben und 30 Hintergrundgesprächen mit lokalen Behörden, Wirtschaftsverbänden und Wissenschaftler*innen zeigt, dass insbesondere klein- und mittelständische Betriebe Schwierigkeiten haben, effektive Maßnahmen zu ergreifen. Kollektive Anpassungsmaßnahmen vom Privatsektor und lokalen Behörden sind bislang wenig implementiert. Basierend auf den Ergebnissen werden abschließend politische Handlungsempfehlungen skizziert, wie das Engagement des Privatsektors gestärkt und besser integriert werden kann.

Schlüsselwörter Risikominderung $\cdot$ Flut $\cdot$ Klimawandel $\cdot$ Jakarta $\cdot$ Ho Chi Minh City $\cdot$ Unternehmen

\begin{abstract}
Ongoing urbanisation and the first effects of climate change are increasing the risk of flooding in coastal Southeast Asian megacities. Local industries are particularly affected in their functionality and competitiveness. At the same time, in the debates on climate change adaptation and disaster risk reduction, the engagement of the private sector in flood protection is discussed, as many local authorities in Southeast Asia cannot substantially reduce flood risk on their own. Against this background, we examine how manufacturing firms in Ho Chi Minh City (Vietnam) and Jakarta (Indonesia) respond to floods, what adaptation measures they implement and the extent to which their engagement contributes to collective adaptation. The analysis of 63 in-depth interviews with manufacturing firms and 30 background discussions with local authorities, business associations and scientists shows that small and medium-sized enterprises in particular have difficulties in taking effective measures. Likewise, collective adaptation measures by the private sector and the local authorities have barely been implemented so far. Finally, based on the findings, policy recommendations on how the engagement of the private sector can be strengthened and better integrated are outlined.
\end{abstract}

Keywords Risk reduction $\cdot$ Floods $\cdot$ Climate change $\cdot$ Jakarta $\cdot$ Ho Chi Minh City $\cdot$ Businesses

Dr. Thomas Neise

thomas.neise@uni-osnabrueck.de

Dr. Roxana Leitold

r.leitold@uni-koeln.de

1 Institut für Geographie, Universität Osnabrück, Seminarstraße 19a/b, 49074 Osnabrück, Deutschland

2 Geographisches Institut, Universität zu Köln, Albertus-Magnus-Platz, 50923 Köln, Deutschland 


\section{Überschwemmungen in südostasiatischen Megastädten - Herausforderungen für den Privatsektor}

Starke Regenfälle und Springtiden verursachen seit Jahrzehnten Überschwemmungen in südostasiatischen Megastädten wie Jakarta in Indonesien und Ho Chi Minh City (HCMC) in Vietnam. Durch die fortschreitende Bebauung und Versiegelung von Grün- und Retentionsflächen sowie der Begradigung von Flüssen können Böden und Flüsse immer weniger Wasser aufnehmen. Unregulierte Grundwasserentnahme und die hohe Flächenauflast des bebauten Bodens verstärken Landabsenkungen, wodurch der Boden im Verhältnis zum Meeresspiegel absinkt und Tidenhochwasser verstärkt auftritt (Bott 2020). Erste Auswirkungen des Klimawandels verstärken die Häufigkeit und das Ausmaß von Überschwemmungen, stellen die Städte, ihre Entscheidungsträger*innen und Bewohner*innen vor vielschichtige Herausforderungen und zeigen die Dringlichkeit, effektive Flutschutzmaßnahmen zu ergreifen.

Prognosen gehen davon aus, dass südostasiatische Megastädte überproportional stark von negativen Klimawandelauswirkungen betroffen sein werden (Hanson et al. 2011; IPCC 2019). Jakarta und HCMC sind hierfür zwei Beispiele, die sich in ihrer topografischen Struktur und ihrer wirtschaftlichen Bedeutung ähneln. Als jeweiliges nationales ökonomisches Zentrum haben deren lokale Überschwemmungen jeweils auch sozioökonomische Folgen. Für andere Landesteile. Große Anteile ihrer Stadtgebiete liegen durch die Küstennähe bis zu $10 \mathrm{~m}$ unter dem Meeresspiegel, was die Gefährdung durch Springtiden, Sturmfluten oder den Meeresspiegelanstieg erhöht. Der durch Landsenkungen verursachte relative Meeresspiegel steigt jährlich um mindestens $4 \mathrm{~cm}$ in Jakarta bzw. im Durchschnitt um 3,3 mm in HCMC (Bott 2020; Duffy et al. 2020). Obgleich nationale und lokale Behörden in diesen Hotspots mit internationaler Unterstützung (u.a. durch die Weltbank und die japanische Entwicklungszusammenarbeit) Fortschritte beim Überschwemmungsschutz erzielt haben, sind die bislang durchgeführten Maßnahmen angesichts der prognostizierten Klimaauswirkungen unzureichend.

Auch das alltägliche Wirtschaften von Unternehmen ist durch wiederkehrende Überschwemmungen beeinträchtigt. Schäden an Produkten, Produktionsanlagen und unterbrochenen Lieferketten sind nur einige Beispiele dafür, wie Überschwemmungen die Wettbewerbsfähigkeit und das langfristige Überleben der Betriebe bedrohen. In HCMC befinden sich circa $25 \%$ und in Jakarta circa $38 \%$ der Industriebetriebe und unzählige (informelle) Kleinstbetriebe in überschwemmungsgefährdeten Gebieten (Leitold 2020; Neise 2019). Insbesondere klein- und mittelständische Unternehmen (KMU) sind in ihrer Betriebsfähigkeit eingeschränkt, da sie häufig in tiefliegenden Küstengebieten lokalisiert sind und von infrastrukturellen Anpassungsmaßnahmen wie Deich- und Schleusensystemen oder dem Ausbau von geschützten Industrieparks kaum profitieren (Leitold und Revilla Diez 2019; Neise 2019).

Gleichzeitig wird der Privatsektor in den wissenschaftlichen und politischen Debatten über Katastrophenrisikominderung und Anpassung an den Klimawandel zunehmend als bedeutender Akteur thematisiert (vgl. IPCC 2019; Linnenluecke et al. 2013; Neise et al. 2018, Schaer und Kuruppu 2018). Insbesondere das „Sendai Framework für Disaster Risk Reduction 2015-2030“ (vgl. UNISDR 2015) hat den Wandel der Katastrophenrisikominderung und der Klimaanpassung zu einem Multistakeholderansatz vorangetrieben und dabei die Verantwortung sowie das Potenzial des Privatsektors betont. Für eine effektive Anpassung wird postuliert, dass Behörden, die Zivilgesellschaft und der Privatsektor ihre Aktivitäten koordiniert vollziehen sollen (Pauw und Chan 2018). Grundsätzlich sollen Unternehmen Investitionen in technische und bauliche Anpassungsmaßnahmen (z.B. Deich- oder Drainagesysteme) mitfinanzieren sowie ihre Expertise einbringen (Johnson und Abe 2015). Gleichzeitig wird argumentiert, dass Unternehmen durch ihr Engagement ihre eigenen betrieblichen Einschränkungen und Schäden reduzieren können (Izumi und Shaw 2015). In der Praxis zeigt sich jedoch, dass dies bislang unzureichend umgesetzt ist. Auffällig ist, dass lokale KMU wenig in die politische Debatte eingebunden sind und Initiativen meistens von multinationalen Unternehmen durchgeführt werden.

Vor diesem Hintergrund untersucht der Artikel, wie betroffene Industriebetriebe auf Überschwemmungen reagieren und welche Maßnahmen sie durchführen, um betriebliche Schäden zu reduzieren. Zudem wird analysiert, ob Industriebetriebe sich an kollektiven Flutschutzmaßnahmen mit anderen Akteur*innen wie lokalen Behörden und der Bevölkerung beteiligen. Um diesen Fragen nachzugehen, werden empirische Ergebnisse aus zwei Dissertationen (Leitold 2020; Neise 2019) zusammengeführt, die Schäden durch Überschwemmungen und betriebliche Anpassungsmaßnahmen in Jakarta und HCMC zwischen 2015 und 2019 untersucht haben.

Das Datenmaterial umfasst 63 Tiefeninterviews mit Betriebsinhaber*innen (vgl. Tab. 1). Um ein vielschichtiges Bild der Betroffenheit zu erhalten, wurden Betriebe unterschiedlicher Größe (gemäß der Angestelltenzahl) und Sektoren (z. B. Nahrungsmittel, Bekleidung, Elektrotechnik) in verschiedenen Stadtvierteln befragt, deren Betriebsfähigkeit in den letzten fünf Jahren mindestens einmal aufgrund von Überschwemmungen beeinträchtigt war. Um den Einfluss von organisatorischen und finanziellen Ressourcen auf das Anpassungsverhalten der Betriebe zu verstehen, wurde die Auswertung der qualitativen Ergebnisse anhand der Betriebsgröße strukturiert. Zusätzlich wurden 30 Hintergrundgespräche (14 Interviews in Jakarta und 16 Interviews 
Tab. 1 Übersicht der interviewten Betriebe gemäß ihrer Angestelltenzahl

\begin{tabular}{lll}
\hline & HCMC & Jakarta \\
\hline Kleinbetriebe (weniger als 50 Angestellte) & 10 & 14 \\
Mittelständische Betriebe (weniger als 250 & 11 & 8 \\
Angestellte) & & \\
Großbetriebe (mehr als 250 Angestellte) & 9 & 11 \\
Summe & 30 & 33 \\
\hline
\end{tabular}

in HCMC) mit politischen Entscheidungsträger*innen, wissenschaftlichen Institutionen, Nichtregierungsorganisationen, lokalen Wirtschaftsverbänden und internationalen Organisationen geführt.

\section{Betroffenheit von Betrieben in HCMC und Jakarta}

Betriebe in HCMC und Jakarta sind in ihrer Arbeitsweise und Funktionsfähigkeit vielfältig durch Überschwemmungen eingeschränkt. Obwohl fast alle befragten Betriebe in HCMC und Jakarta von indirekten Schäden durch überflutete Straßen (vgl. Abb. 1) wie unterbrochenen Lieferketten und verzögertem Transport betroffen sind, wird deutlich, dass KMU, die für den lokalen Markt produzieren und nicht in internationale Lieferketten eingebunden sind, die Hauptlast der Schäden tragen. Mehr als jedes zweite befragte KMU in Jakarta und HCMC erlebt regelmäßig direkte Schäden an Gebäuden, Produktionsanlagen oder Fahrzeugen. Nicht sachgemäß gelagertes Material und produzierte Güter werden schon von wenigen Zentimetern Hochwasser in der Produktionsstätte beschädigt. Zudem berichteten KMU von weitreichenden Produktionsunterbrechungen, die aufgrund regelmäßiger Überschwemmungen durch Stürme und starke Regenfälle beispielsweise in HCMC bis zu 30 Tage pro Jahr andauern. In Jakarta müssen die KMU ihre Produktion einstellen bzw. reduzieren, da es zu Stromausfällen kommt. Die Betriebe müssen häufig mit weniger Personal auskommen, weil Straßen unpassierbar sind und Arbeitskräfte ihr privates Eigentum schützen müssen. Durch die Produktionsunterbrechung und die eingeschränkte Versorgung mit Rohmaterial durch Lieferverzögerungen ist die Nichterfüllung von Produktions- und Lieferverträgen in beiden Städten ein Problem für ansässige Betriebe.

Großbetriebe in HCMC produzieren hingegen häufig in geschützten Industrieparks in See- und Flughafennähe. Obwohl auch hier zeitweilig Personalknappheit durch lokale Überschwemmungen herrscht, werden indirekte Flutschäden als gering eingestuft, da die Infrastruktur außerhalb der Industriezonen kaum bis wenig genutzt wird. Moderne Abfluss- und Pumpsysteme und lokal erhöhte Straßen in Industrieparks bieten den Produktionsstandorten der Betrie- be einen bislang ausreichenden Flutschutz. In Jakarta sind Großbetriebe ebenfalls durch eigene Pumpstationen, Retentionsbecken und höhergelegte Produktions- und Stromversorgungsstätten geschützt (siehe Abb. 2).

\section{Anpassungsmaßnahmen von Industriebetrieben}

Unternehmensspezifische Charakteristika und externe Umfeldfaktoren führen zu unterschiedlichen Ausprägungen von Anpassungsmaßnahmen des produzierenden Gewerbes (Neise und Revilla Diez 2019; Leitold 2020; vgl. Abb. 3). Viele Betriebe setzen ihre Maßnahmen allein, teils aber auch in Zusammenarbeit mit anderen Unternehmen und der Nachbarschaft um. Vor allem exportorientierte, kapitalund ressourcenstarke Großbetriebe in HCMC und Jakarta sind gut auf Überschwemmungen vorbereitet und widmen sich der langfristig angelegten Vorsorge - und dass, obwohl sie bislang kaum direkt betroffen sind. Ihr Engagement beruht nicht primär auf den Entscheidungen des lokalen Managements, sondern die Entscheidungsgewalt obliegt oft den ausländischen Unternehmenszentralen. So berichtete ein Baumaschinenhersteller in Jakarta, dass das japanische Hauptquartier Investitionen in den Flutschutz (beispielsweise Schutzmauern und Pump- bzw. Drainagesysteme) und das Training des Personals anordnete. Auf die geringen indirekten Auswirkungen auf die Lieferketten reagieren die Betriebe mit zeitlichen und organisatorischen Anpassungen innerhalb des Lieferprozesses.

In Jakarta haben Flutkatastrophen, wie beispielsweise im Jahr 2007, zu einem Umdenken bei den Betrieben geführt. Nachdem mehr als die Hälfte der Stadt über Wochen überschwemmt war und hohe direkte Schäden auftraten, haben Großbetriebe kontinuierlich ihre betrieblichen Routinen verändert und neue Kompetenzen, wie die Einführung eines Betriebskontinuitätsmanagements und eines Frühwarnsystems, erworben:

Nach 2007 haben wir eine Menge Verbesserungen vorgenommen, weil wir aus den Erfahrungen der schrecklichen Überschwemmungen gelernt haben. Wir haben ... die Mauern verstärkt, unser Gebäude und das Firmengelände erhöht und die Kapazität der Wasserpumpen sowie die Wasserkanäle erweitert (Aussage von einem Manager eines Automobilherstellers in Jakarta, eigene Übersetzung).

Eine Standortverlagerung aus überschwemmungsgefährdeten Gebieten wird von den Unternehmen sowohl in HCMC als auch in Jakarta nicht als Lösung präferiert. Mangelnde Alternativstandorte, hohe Kosten für Verlagerungen und die soziale Verankerung innerhalb der Stadtviertel sind ausschlaggebend für das Festhalten am bisherigen Standort. 


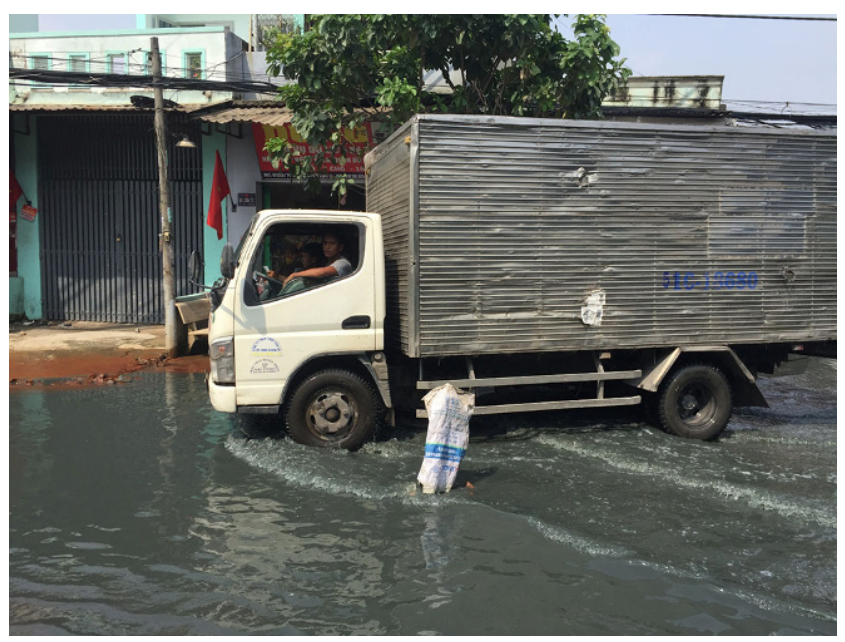

Abb. 1 Überschwemmte Straßen in HCMC durch zeitgleich auftretende Springtiden und Starkregenfälle (Foto: Roxana Leitold)

Allerdings müssen immer mehr Betriebe ihren innerstädtischen Produktionsstandort aufgeben, da die Produktionserlaubnis nicht verlängert wird. Die Behörden betrachten in Wohnviertel integrierte Industriebetriebe als Verursacher von Umweltverschmutzung und Überschwemmungen. Daher hat eine Umsiedlung von Industriebetrieben in die Vororte von Jakarta im letzten Jahrzehnt begonnen. Insbesondere informelle Betriebe beispielsweise in der Nahrungsmittelproduktion waren häufig an Flussufern ansässig, von wo sie als Folge der behördlichen Politik, die Flussbetten auszuweiten, verdrängt wurden. In HCMC lassen sich ähnliche Tendenzen feststellen. Hier soll vor allem in den nächsten Jahren die Umsiedlung von Industriebetrieben in Gewerbegebiete außerhalb der Kernstadt forciert werden.
Obwohl KMU in HCMC und Jakarta bereits heute stark von Überschwemmungen betroffen sind, fehlen langfristige und strategische Betriebsplanungen. Während der Regensaison verlagern kleinere Betriebe beispielsweise ihre Maschinen und Produkte temporär an höhergelegene Stellen und benutzen während der Überschwemmungen kleine Pumpen und Sandsäcke, um das Wasser fernzuhalten. Zeitgleich werden Produktionsausfälle während einer Überschwemmung in Kauf genommen. Drei Gründe sind hierfür ursächlich: Einerseits sind Kleinbetriebe mit vielfältigen Betriebsrisiken wie Arbeitskräftemangel, hohen Inflationsraten und erhöhtem Wettbewerb konfrontiert, die es erschweren, finanzielle Mittel in den Flutschutz zu investieren. Obwohl KMU das Rückgrat der Wirtschaft darstellen, sind die institutionellen Rahmenbedingungen zugunsten von multinationalen und (in Vietnam) staatlichen Unternehmen ausgelegt. Viele KMU sind immer noch mit einem begrenzten Zugang zu Krediten und ineffektiver staatlicher Unterstützung konfrontiert. Viele Betriebe mit begrenztem Kapital sind der Ansicht, dass vornehmlich staatliche und lokale Behörden für den Flutschutz zuständig sind und vertrauen daher auf großangelegte Maßnahmen wie lokale Deich- und Entwässerungssysteme. Allerdings profitieren die Betriebe bislang wenig von staatlichen Flutschutzmaßnahmen, was auf Kritik bei den Betrieben stößt:

Solange ich hier lebe, habe ich noch nie gesehen, dass die Regierung den Fluss ausgräbt oder die Straße anhebt. ... Bis heute gab es keine Aktivitäten der Regierung (Aussage einer Inhaberin eines Bekleidungsherstellers in Jakarta, eigene Übersetzung).

Zudem lässt sich bei KMU, welche seit Jahrzehnten in überschwemmungsgefährdeten Gebieten ansässig sind, ein un-
Abb. 2 Auswahl von Anpassungsmaßnahmen: a höher gebaute Stromversorgungsstation; b Pumpsystem eines Betriebs und höher gelegte Einfahrt; c gereinigter und erweiterter Abflusskanal (Fotos: Roxana Leitold und Thomas Neise)
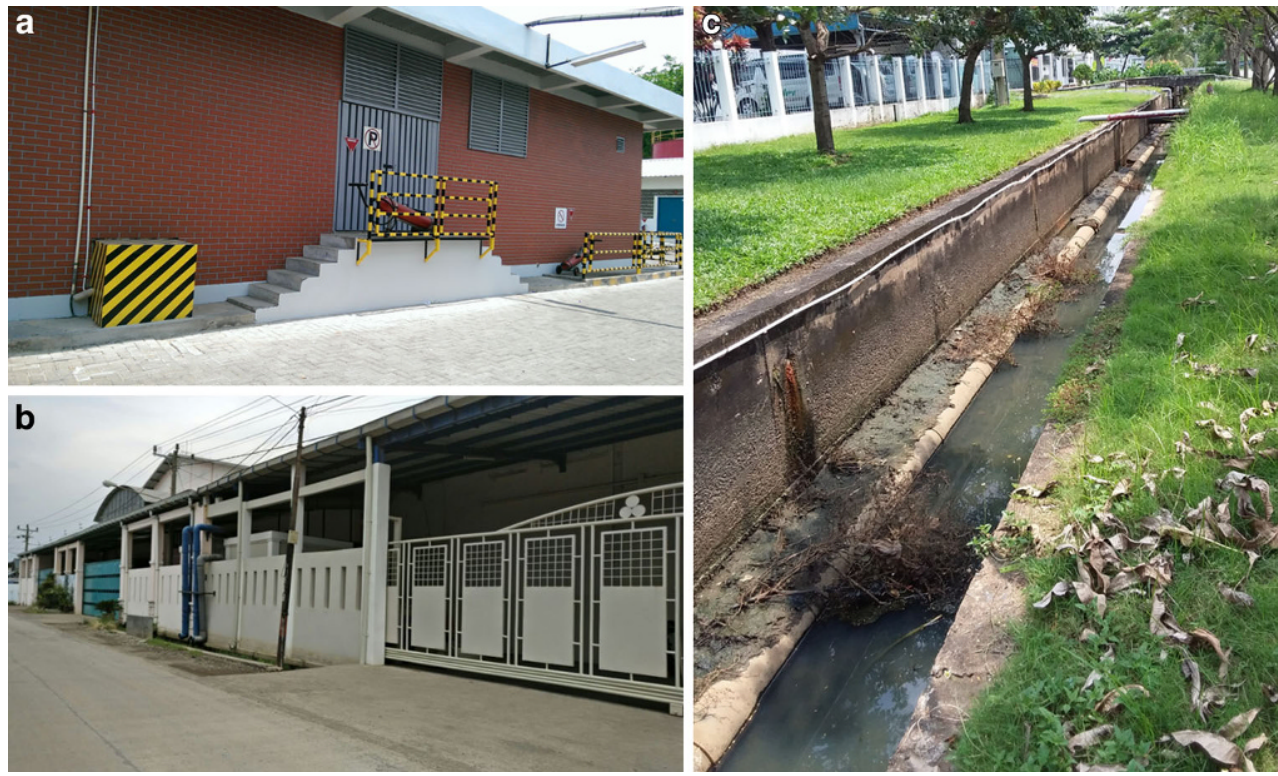


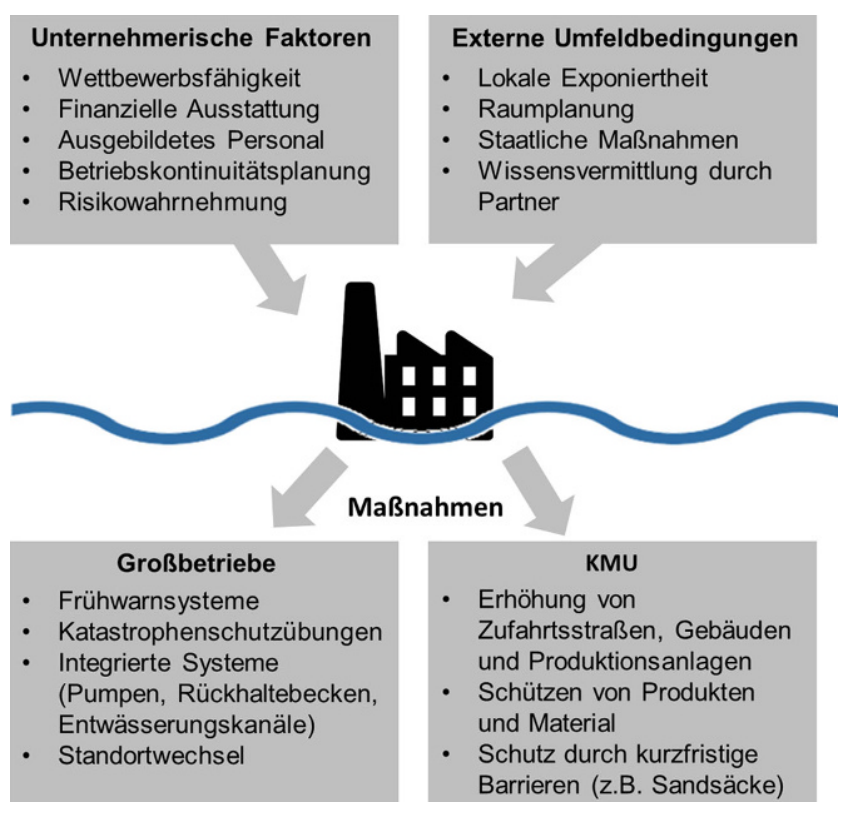

Abb. 3 Treiber und Maßnahmenbeispiele von betrieblicher Anpassung an Überschwemmungen

zureichendes Bewusstsein gegenüber der Gefährdung erkennen. Diese Betriebe nehmen Überschwemmungen oft als gewöhnliches Ereignis wahr und planen daher häufig keine langfristigen Maßnahmen.

\section{Begrenzte Wirkung von unternehmerischen Anpassungsmaßnahmen}

Die Fallbeispiele zeigen, dass nur ein Teil der Industriebetriebe eigene Maßnahmen ergreift, um betriebliche Schäden und Einschränkungen fernzuhalten. Trotz der vielfältigen Anpassungsmaßnahmen wird deutlich, dass die meisten Betriebe eigenständig nicht in der Lage sind, ihr Überschwemmungsrisiko fundamental zu senken. Um die Situation zu verbessern, haben sich beispielsweise Betriebe in einigen Vierteln von Jakarta mit der Bevölkerung und den lokalen Behörden zusammengeschlossen. Gemeinsam wird eine zentrale Pumpstation betrieben und die Entwässerungskanäle gereinigt und erweitert. In HCMC installieren Unternehmer*innen und Anwohner*innen in besonders betroffenen Vierteln gemeinsam kleinere Pumpen und bauen während der Regenzeit Schutzmauern aus Sandsäcken. Solche positiven Beispiele kollektiver Anpassungsmaßnahmen bleiben jedoch Ausnahmen. Es überwiegt die Umsetzung individueller Maßnahmen, welche nicht mit infrastrukturellen Bemühungen und strategischen Maßnahmen der Behörden koordiniert werden. Einige Unternehmen in HCMC und Jakarta berichteten, dass die Auswirkungen von Überschwemmungen gar zugenommen haben, seitdem Haupt- straßen in ihren Viertel von behördlicher Seite angehoben wurden.

Die Umsetzung einer holistischen stadtweiten Flutanpassungsstrategie bleibt daher eine Herausforderung. Kritisch ist insbesondere die Lage von KMU, welche zukunftsorientierte und strategische Anpassungsmaßnahmen kaum bis gar nicht umsetzen und wenig von den bisherigen infrastrukturellen Maßnahmen der Behörden profitieren:

Ja, die Regierung ist verantwortlich, aber im Moment sehe ich ihre Rolle nicht. Normalerweise beauftragt die Regierung eine Firma, die mit einer großen Pumpe Wasser aus den überfluteten Straßen im Stadtzentrum beseitigt. Das ist nicht wirklich effektiv, weil sie nur den Bereich der reichen Menschen unterstützen (Aussage eines Unternehmers von Stahlprodukten in HCMC, eigene Übersetzung).

\section{Stärkere Förderung von KMU - Was können Behörden tun?}

Um die zukünftige Risikoanpassung insbesondere von KMU in den skizzierten Kontexten der Megastädte zu fördern, sollten lokale Behörden KMU stärker für zukünftige Risiken und die Dringlichkeit von effektiveren Anpassungsmaßnahmen sensibilisieren. Bislang greifen viele Betriebe kaum auf öffentliche Warnsysteme vor starken Regenfällen und Springtiden zurück, um präventiv Schutzmaßnahmen vorzunehmen. Zudem sollten KMU eine individuelle Beratung angeboten sowie Anreize geschaffen werden, damit sie effektivere und nachhaltigere Anpassungsstrategien umsetzen. Vor allem wenn KMU nicht in Partnerschaften zwischen lokalen Produzent*innen und multinationalen Unternehmen eingebunden sind, bedarf es eines intensiveren Wissenstransfers zwischen den KMU und den zuständigen Lokalbehörden. Wirtschaftsverbände oder Handelskammern können diese wichtige Rolle auf lokaler Ebene einnehmen und KMU über mögliche Anpassungsinitiativen sowie Finanzierungsmöglichkeiten schulen und beraten. In diesem Zusammenhang sollten zweckgebundene Finanzmittel für Anpassungsmaßnahmen mobilisiert sowie robuste Versicherungssysteme für Naturrisiken aufgebaut werden. In beiden Ländern gibt es kaum Versicherungsmodelle für Unternehmen bzw. die Prämien sind insbesondere für KMU zu hoch. Daher gilt es, die politischen Voraussetzungen für den Aufbau privater Versicherungslösungen voranzutreiben.

Kollektive Anpassungsmaßnahmen sollten zudem stärker gefördert werden. Das Gespräch mit der Katastrophenschutzbehörde in Jakarta ergab, dass Ko-Finanzierungen von Flutschutzmaßnahmen gesetzlich nicht umsetzbar sind. Somit bleibt das Potenzial der Kooperation von Unterneh- 
men und Katastrophenschutzbehörden ungenutzt. Weiterführende Projektstudien zeigen, dass viele befragte KMU in HCMC und Jakarta bereit sind, sich gemeinsam mit den lokalen Behörden und der Bevölkerung an Flutanpassungsmaßnahmen zu beteiligen (vgl. Leitold et al. 2020; Neise et al. 2019). Daher sollten die Potenziale von Multistakeholderansätzen auch in den rechtlichen Rahmenbedingungen zur Anpassung an Überschwemmungen - und Klimaauswirkungen generell - stärker berücksichtigt werden. Schlussendlich können die Akteur*innen in den küstennahen Megastädten Südostasiens das (zukünftige) Überschwemmungsrisiko nur gemeinsam bewältigen und somit die Wettbewerbsfähigkeit der Betriebe und die zukunftsfähige Entwicklung der Städte vorantreiben.

Förderung Die Forschung wurde unterstützt vom Bundesministerium für Bildung und Forschung (BMBF) im Rahmen des DECIDERProjekts (Förderkennzeichen: 01LZ1703D) sowie der Deutschen Forschungsgesellschaft im Rahmen der Exzellenzinitiative.

Funding Open Access funding enabled and organized by Projekt DEAL.

Open Access Dieser Artikel wird unter der Creative Commons Namensnennung 4.0 International Lizenz veröffentlicht, welche die Nutzung, Vervielfältigung, Bearbeitung, Verbreitung und Wiedergabe in jeglichem Medium und Format erlaubt, sofern Sie den/die ursprünglichen Autor(en) und die Quelle ordnungsgemäß nennen, einen Link zur Creative Commons Lizenz beifügen und angeben, ob Änderungen vorgenommen wurden.

Die in diesem Artikel enthaltenen Bilder und sonstiges Drittmaterial unterliegen ebenfalls der genannten Creative Commons Lizenz, sofern sich aus der Abbildungslegende nichts anderes ergibt. Sofern das betreffende Material nicht unter der genannten Creative Commons Lizenz steht und die betreffende Handlung nicht nach gesetzlichen Vorschriften erlaubt ist, ist für die oben aufgeführten Weiterverwendungen des Materials die Einwilligung des jeweiligen Rechteinhabers einzuholen.

Weitere Details zur Lizenz entnehmen Sie bitte der Lizenzinformation auf http://creativecommons.org/licenses/by/4.0/deed.de.

\section{Literatur}

Bott L-M (2020) Living with sea level change and coastal flooding Collective responses of households and communities in Indonesia. Universität zu Köln, Köln

Duffy CE, Braun A, Hochschild V (2020) Surface subsidence in urbanized coastal areas: PSI methods based on sentinel-1 for ho chi Minh city. Remote Sens 12(24):4130. https://doi.org/10.3390/ rs 12244130
Hanson S et al (2011) A global ranking of port cities with high exposure to climate extremes. Clim Change 104:89-111. https://doi. org/10.1007/s10584-010-9977-4

IPCC (2019) Special report on the ocean and Cryosphere in a changing climate. IPCC, Genf

Izumi T, Shaw R (2015) Overview and introduction of the private sector's role in disaster management. In: Izumi T, Shaw R (Hrsg) Disaster management and private sectors. Springer, Tokyo, S 1-10

Johnson DAK, Abe Y (2015) Global overview on the role of the private sector in disaster risk reduction: scopes, challenges, and potentials. In: Izumi T, Shaw R (Hrsg) Disaster management and private sectors. Springer, Tokyo, S 11-29

Leitold R (2020) Private sector engagement in flood risk reduction and climate change adaptation - Insights from manufacturing firms in Ho Chi Minh City, Vietnam. Dissertation. Universität zu Köln, Köln

Leitold R, Revilla Diez J (2019) Exposure of manufacturing firms to future sea level rise in Ho Chi Minh City, Vietnam. J Maps 15:13-20. https://doi.org/10.1080/17445647.2018.1548385

Leitold R, Revilla Diez J, Tran V (2020) Are we expecting too much from the private sector in flood adaptation? Scenario-based field experiments with small- and medium-sized firms in Ho Chi Minh City, Vietnam. Clim Change 163:359-378. https://doi.org/10. 1007/s10584-020-02888-y

Linnenluecke MK, Griffiths A, Winn MI (2013) Firm and industry adaptation to climate change: a review of climate adaptation studies in the business and management field. Wires Clim Chang 4:397-416. https://doi.org/10.1002/wcc. 214

Neise T (2019) Firms' adaptation strategies to floods and their potential implication on regional economic development-insights from Jakarta and Semarang, Indonesia. Dissertation. Universität zu Köln, Köln

Neise T, Revilla Diez J (2019) Adapt, move or surrender? Manufacturing firms' routines and dynamic capabilities on flood risk reduction in coastal cities of Indonesia. Int J Disaster Risk Reduct 33:332-342. https://doi.org/10.1016/j.ijdrr.2018.10.018

Neise T, Revilla Diez J, Garschagen M (2018) Firms as drivers of integrative adaptive regional development in the context of environmental hazards in developing countries and emerging economies-a conceptual framework. Environ Plan C Polit Space 36:1522-1541. https://doi.org/10.1177/2399654418771079

Neise T, Sambodo MT, Revilla Diez J (2019) Are micro-, small- and medium-sized enterprises willing to contribute to collective flood risk reduction? Scenario-based field experiments from jakarta and Semarang, Indonesia. Organ Environ. https://doi.org/10. $1177 / 1086026619875435$

Pauw WP, Chan S (2018) Multistakeholder partnerships for adaptation. The role of micro, small and medium enterprises. In: Schaer C, Kuruppu N (Hrsg) Private-sector action in adaptation: perspectives on the role of micro, small and medium size enterprises. UNEP DTU Partnership, Copenhagen, S 99-109

Schaer C, Kuruppu N (2018) Private-sector action in adaptation: perspectives on the role of micro, small and medium size enterprises. UNEP DTU Partnership, Copenhagen

UNISDR (2015) Sendai framework for disaster risk reduction 2015-2030. UNISDR, Genf 